LA WAE NCE LIWEAMCAE NATIONAL LABDAATOAY
Rotational bands and isomeric states in ${ }^{175} \mathrm{Lu}$
P.E. Garrett, D.E. Archer, J.A. Becker, L.A. Bernstein, K. Hauschild, E.A. Henry, D.P. McNabb, M.A. Stoyer, W. Younes, G.D. Johns, R.O. Nelson, W.S. Wilburn

October 1, 2003 


\section{DISCLAIMER}

This document was prepared as an account of work sponsored by an agency of the United States Government. Neither the United States Government nor the University of California nor any of their employees, makes any warranty, express or implied, or assumes any legal liability or responsibility for the accuracy, completeness, or usefulness of any information, apparatus, product, or process disclosed, or represents that its use would not infringe privately owned rights. Reference herein to any specific commercial product, process, or service by trade name, trademark, manufacturer, or otherwise, does not necessarily constitute or imply its endorsement, recommendation, or favoring by the United States Government or the University of California. The views and opinions of authors expressed herein do not necessarily state or reflect those of the United States Government or the University of California, and shall not be used for advertising or product endorsement purposes.

This work was performed under the auspices of the U. S. Department of Energy by the University of California, Lawrence Livermore National Laboratory under Contract No. W-7405-Eng-48. 


\title{
Rotational bands and isomeric states in ${ }^{175} \mathrm{Lu}$
}

\author{
P.E. Garrett, D.E. Archer, J.A. Becker, L.A. Bernstein, K. \\ Hauschild, [†] E.A. Henry, $\left.{ }^{*}\right]$ D.P. McNabb, M.A. Stoyer, W. Younes \\ Lawrence Livermore National Laboratory, Livermore, CA 94551 \\ G.D. Johns, R.O. Nelson, W.S. Wilburn \\ Los Alamos National Laboratory, Los Alamos, NM 87545
}

\begin{abstract}
Rotational bands in ${ }^{175} \mathrm{Lu}$ have been extended through investigation with the $\left(n, n^{\prime} \gamma\right)$ reaction. Spallation neutrons bombarded Lu samples, and the resulting $\gamma$ rays were detected in a large-scale Compton-suppressed Ge detector array. Prompt- and delayed- $\gamma \gamma$ coincidences have been used to extend most of the existing known bands, and to tentatively assign a new band, based on the $7 / 2^{-}[523]$ configuration, from its band head to spin $13 / 2$. The 3 -quasiparticle $K^{\pi}=19 / 2^{+}$isomer is confirmed and its half life determined to be $984 \pm 13$ (stat.) \pm 30 (sys.) $\mu$ s, in agreement with previous results.
\end{abstract}


There has been a renewed interest in isomeric states in the Hf region recently, in part due to reports [1] of stimulated emission from the $31 \mathrm{y}, 16^{+}$isomer in ${ }^{178} \mathrm{Hf}$. While the result [1] has been refuted [2], the physics interest in the high- $K$ multi-quasiparticle isomers in the region is driven by the quest to understand the purity of the $K$ quantum number and its persistence to high excitation energy. Nuclei on the neutron-rich side of the valley of stability are a promising laboratory for isomer studies, and access to these nuclei has recently become accessible through fragmentation reactions (e.g. Ref.[3]) or deep inelastic collisions. Systematic investigations that study the evolution of intrinsic excitations as a function of proton and neutron number are required in order to make firm configuration assignments and to assess the $K$ purity. It is the purpose of this Brief Report to communicate results of measurements taken on ${ }^{175} \mathrm{Lu}$ with neutrons originating from a spallation neutron source. In these experiments, rotational bands have been extended based on analysis of promptand delayed $\gamma \gamma$ coincidences, a new rotational band built on the $7 / 2^{-}[523]$ configuration is suggested, and the known [4] three-quasiparticle 19/2+ isomer has been confirmed and its lifetime re-measured. While the lack of statistics and the spectral complexity has prevented the detailed study of the band properties, the present results are useful to future detailed studies of this nucleus.

The measurements were performed at the Los Alamos Neutron Science Center (LANSCE) Weapons Neutron Research facility. Spallation neutrons were produced by bombarding a ${ }^{\text {nat }} \mathrm{W}$ target with $800 \mathrm{MeV}$ protons from the LANSCE linac. The pulsed proton beam was delivered with a $1.8 \mu$ s spacing for $625 \mu$ s macropulses at a macropulse rate of (typically) $80 \mathrm{~Hz}$. The scattering sample consisted of two 3-mm-thick pieces of ${ }^{n a t} \mathrm{Lu}_{2} \mathrm{O}_{3}$ loaded VCE plastic with weight percents of $69.57 \% \mathrm{Lu}$ (with a natural abundance of $97.4 \%{ }^{175} \mathrm{Lu}$ ), $14.20 \%$ $\mathrm{O}, 15.77 \% \mathrm{C}, 0.20 \% \mathrm{H}$, and $0.26 \% \mathrm{~N}$. The sample was placed at the focus of the GEANIE spectrometer located at a distance of $20.34 \mathrm{~m}$ from the production target on the $60^{\circ} \mathrm{R}$ flight path. The neutrons were collimated to produce a 1.5-cm-diameter (FWHM) "beam" at the scattering sample. In order to reduce the number of low-energy neutrons and to reduce the intensity of $\gamma$ rays from the neutron production target, $7.5 \mathrm{~cm}$ of polyethylene and 2.9 $\mathrm{cm}$ of $\mathrm{Pb}$ were placed in the neutron beam $14 \mathrm{~m}$ upstream of the scattering sample. The 
GEANIE spectrometer consisted of 11 planar and 15 25\% HPGe coaxial detectors, whose front faces were located $14 \mathrm{~cm}$ from the scattering sample. All planar and 9 of the coaxial detectors were equipped with suppression shields. The planar detectors were concentrated at the most forward and backward angles, and $\gamma$-ray events of $\leq 1 \mathrm{MeV}$ were processed, while the coaxial detectors were positioned around $90 \pm 40^{\circ}$ with respect to the beam direction and $\gamma$-ray events were recorded with energies up to $4 \mathrm{MeV}$. The data were collected in singles-and-higher-fold mode. The time relative to the start of the macropulse (CLOCK, recorded in $100 \mathrm{~ns}$ intervals), the energy $E_{\gamma}$ and, if in-beam, the time $t_{\gamma}$ relative to the proton micropulse were collected for each detector that indicated an event. The neutron flux was monitored using a fission chamber with both ${ }^{235} \mathrm{U}$ and ${ }^{238} \mathrm{U}$ foils [5].

During playback of the data, events were separated depending on whether they occurred during the macropulse (in-beam) or after (out-of-beam). Matrices, including $E_{\gamma}$ vs. timeof-flight (TOF) and $\gamma \gamma$ coincidences, both prompt and delayed, were created. The prompt $\gamma \gamma$ coincidences were created by placing a time condition that events occur within $\approx 100$ ns of each other and correspond to $E_{n}=1-8 \mathrm{MeV}$. A total of $2.6 \times 10^{6}$ events were sorted into the prompt matrix. The delayed $\gamma \gamma$ coincidence matrix had the condition that the times between events be in the range of $150-750 \mathrm{~ns}$, with the further condition that they correspond to $E_{n}<600 \mathrm{MeV}$. The out-of-beam data were sorted into $E_{\gamma} v s$. CLOCK and $\gamma \gamma$ matrices. Additional details of the experimental setup and data analysis are given in Ref. [6].

The 1.49(7) $\mu$ s isomer [7], located at $353 \mathrm{keV}$, decays by a $353-\mathrm{keV} E 1$ transition from the $5 / 2^{-}$member of the $1 / 2^{-}[541]$ configuration to the ground state, which has the $7 / 2^{+}[404]$ configuration. The spectrum of delayed coincidences with the 353-keV transition, shown in Fig. 1, displays transitions that are emitted $150-750 \mathrm{~ns}$ before the $353-\mathrm{keV}$ transition, i.e. these $\gamma$ rays feed levels located above the $353-\mathrm{keV}$ isomeric level. Most of the transitions have been placed previously $[8,9]$ in the ${ }^{175} \mathrm{Lu}$ level scheme. Some additional transitions are observed, and from Fig. 1 and results of gates placed on the prompt $\gamma \gamma$ matrix, allow the extension of the $1 / 2^{+}[411]$ and $1 / 2^{-}[541]$ bands to higher excitation energy as shown in Fig. 2. The $15 / 2^{+}$and $21 / 2^{-}$members of these bands, observed by Foin et al. [8], were 
not adopted in the most recent Nuclear Data Sheets [10]. The present work confirms the existence of these levels. Three new levels, at $1311.2 \mathrm{keV}, 1434.1 \mathrm{keV}$, and $1573.4 \mathrm{keV}$, are established based on prompt $\gamma \gamma$ coincidence results. The decay patterns to the $7 / 2^{+}, 9 / 2^{+}$, and $11 / 2^{+}$levels of the $1 / 2^{+}[411]$ band, and the very good agreement with the level energies predicted based on a rotational parameter of $13.6 \mathrm{keV}$ extracted from the $5 / 2^{+}-3 / 2^{+}$energy difference, supports the assignment of these levels as the $7 / 2^{+}, 9 / 2^{+}$, and $11 / 2^{+}$members of the $3 / 2^{+}[411]$ band tentatively assigned in Ref. [9]. Also shown is the extension of the $5 / 2^{+}[402]$ band to $17 / 2^{+}$based on $M 1 / E 2$ in-band transitions, and the assignment of the $21 / 2^{-}$band member of the $9 / 2^{-}[514]$ band. Table I lists the relative $\gamma$-ray intensities for each level where it was possible to extract the uncontaminated peak areas from the coincidence gates.

Shown in Fig. 3 is the spectrum obtained from summing gates of $396 \mathrm{keV}$ and $282 \mathrm{keV}$ placed on the prompt $\gamma \gamma$ coincidence matrix. In addition to the in-band members, $\gamma$ rays with energies of $609 \mathrm{keV}, 583 \mathrm{keV}, 557 \mathrm{keV}, 713 \mathrm{keV}, 716 \mathrm{keV}$, and $530 \mathrm{keV}$ are observed. These transitions are placed as is illustrated Fig. 2. With dipole or quadrupole transitions, the spin of the $1005-\mathrm{keV}$ level is limited to the range 5/2-13/2. Assuming that the levels established by the above listed $\gamma$ rays form a rotational band, and that the $1005-\mathrm{keV}$ level is the band head, rotational-model fits were performed for different values of $I$ and $K$. A very good fit was found for an $I(I+1)$ sequence with $K=7 / 2$ and a rotational parameter of $11.9 \mathrm{keV}$ from the $1112-1005 \mathrm{keV}$ energy difference, as shown in Fig. 4. The lack of any observed transitions to other bands, the absence of stretched E2 transitions and the (presumably) dipole nature of the transitions to the $9 / 2^{-}[514]$ band favors a $7 / 2^{-}[523]$ configuration assignment. The suggestion of the $1005-\mathrm{keV}$ level as the $7 / 2^{-}[523]$ band head fits in perfectly with the trend in excitation energy observed with increasing $A$ for the $7 / 2^{-}[523]$ orbital in the lighter $\mathrm{Lu}$ isotopes.

Based on results [4] from inelastic collisions of a ${ }^{238} \mathrm{U}$ beam on a ${ }^{175} \mathrm{Lu}$ target, an isomer at $1391 \mathrm{keV}$ in ${ }^{175} \mathrm{Lu}$ was located and measured to have a half life of $930(80) \mu$ s. This level was known from earlier ${ }^{176} \mathrm{Lu}(d, t)$ reactions studies [11], where it was assigned as the $K^{\pi}=19 / 2^{+}, 7 / 2^{+}[404]_{\pi} \otimes 7 / 2^{-}[514]_{\nu} \otimes 5 / 2^{-}[512]_{\nu}$ 3-quasiparticle state, and was also 
excited in the present work. Shown in Fig. 5 is a singles spectrum obtained during the outof-beam period where a spectrum gated on the event times $3.375<t_{\gamma}(\mathrm{ms})<6.575$ after a macropulse is subtracted from a spectrum gated on the event times $0.175<t_{\gamma}(\mathrm{ms})<3.375$. This subtraction of the two time-gated spectra has the effect of removing the long-lived $\beta$ decay lines from the spectrum. The remaining $\gamma$ rays belong to the $19 / 2^{+}$isomer decay path or are other well-known, neutron-induced, background lines. Figure 6 displays the decay curve of the 797-keV E2 transition; a half life of 984(13) $\mu$ s is extracted after a timedependent dead time correction was applied. The uncertainty does not include an estimated $\approx 30 \mu$ s systematic uncertainty due to this dead time correction. The present measured half life is in agreement with, but more precise than, the measurement of Wheldon et al. [4] of 930(80) $\mu \mathrm{s}$.

In summary, rotational bands and isomeric levels in ${ }^{175} \mathrm{Lu}$ have been observed with the $\left(n, n^{\prime} \gamma\right)$ reaction using spallation neutrons. An analysis of the prompt and delayed $\gamma \gamma$ coin-

cidence matrices confirm and extend the previous level schemes for the $5 / 2^{+}[402], 3 / 2^{+}[411]$, $1 / 2^{+}[411], 1 / 2^{-}[541]$, and $9 / 2^{-}[514]$ rotational bands. A new rotational band, suggested to have the $7 / 2^{-}[523]$ configuration, is assigned. The 3 -quasiparticle $K^{\pi}=19 / 2^{+}$isomer is observed, and its half life measured to be $984 \pm 13$ (stat.) \pm 30 (sys.) $\mu$ s.

This work was performed under the auspices of the U.S. Department of Energy by The University of California, Lawrence Livermore National Laboratory and Los Alamos National Laboratory under contract no. W-7405-ENG-48 and W-7405-ENG-36.

\section{REFERENCES}

[†] Present address: CSNSM, IN2P3-CNRS, F-91405 Orsay, Cedex, France.

[*] Present address: Office of Nuclear Physics, SC-90/Germantown Building, U.S. Department of Energy, 1000 Independence Ave. SW, Washington, D.C. 20585-1290

[1] C.B. Collins, F. Davanloo, M.C. Iosif, R. Dussart, J.M. Hicks, S.A. Karamian, C.A. Ur, I.I. Popescu, V.I. Kirischuk, J.J. Carroll, H.E. Roberts, P. McDaniel, C.E. Crist, Phys. Rev. Lett. 82, 695 (1999); ibid, Phys. Rev. Lett. 84, 2541 (2000).

[2] I. Ahmad, J.C. Banar, J.A. Becker, D.S. Gemmell, A. Kraemer, A. Mashayekhi, D.P. 
McNabb, G.G. Miller, E.F. Moore, L.N. Pangault, R.S. Rundberg, J.P. Schiffer, S.D. Shastri, T.F. Wang, J.B. Wilhelmy, Phys. Rev. Lett. 87, 072503 (2001).

[3] Zs. Podolyak, P.H. Regan, M. Pfutzner, J. Gerl, M. Hellstrom, M. Caamano, P. Mayet, Ch. Schlegel, A. Aprahamian, J. Benlliure, A.M. Bruce, P.A. Butler, D. Cortina Gil, D.M. Cullen, J. Döring, T. Enqvist, F. Rejmund, C. Fox, J. Garces Narro, H. Geissel, W. Gelletly, J. Giovinazzo, M. Gorska, H. Grawe, R. Grzywacz, A. Kleinbohl, W. Korten, M. Lewitowicz, R. Lucas, H. Mach, M. Mineva, C.D. O'Leary, F. De Oliveira, C.J. Pearson, M. Rejmund, M. Sawicka, H. Schaffner, K. Schmidt, Ch. Theisen, P.M. Walker, D.D. Warner, C. Wheldon, H.J. Wollersheim, S.C. Wooding, F.R. Xu, Phys. Lett. B491, 225 (2000).

[4] C. Wheldon, R. D'Alarcao, P. Chowdhury, P.M. Walker, E. Seabury, I. Ahmad, M.P. Carpenter, D.M. Cullen, G. Hackman, R.V.F. Janssens, T.L. Khoo, D. Nisius, C.J. Pearson, and P. Reiter, Phys. Lett. B425, 239 (1998).

[5] S.A. Wender, S. Balestrini, A. Brown, R.C. Haight, C.M. Laymon, T.M. Lee, P.W. Lisowski, W. McCorkle, R.O. Nelson, W. Parker, and N.W. Hill, Nucl. Instrum. Methods Phys. Res. A336, 226 (1993).

[6] P.E. Garrett, W. Younes, J.A. Becker, L.A. Bernstein, E.M. Baum, D.P. DiPrete, R.A. Gatenby, E.L. Johnson, C.A. McGrath, S.W. Yates, M. Devlin, N. Fotiades, R.O. Nelson, and B.A. Brown, Phys. Rev. C 68, 024312 (2003).

[7] K.H. Johansen, B. Bengtson, P.G. Hansen, P. Hornshoj, Nucl. Phys. A133, 213 (1969).

[8] C. Foin, S. André, and S.A. Hjorth, Nucl. Phys. A219, 347 (1974).

[9] G. Winter, W. Andrejtscheff, L. Funke, P. Manfrass, and H. Sodan, Nucl. Phys. A223, 320 (1974).

[10] A.O. Macchiavelli and E. Browne, Nucl. Data Sheets, 69, 903 (1993).

[11] M. Minor, R.K. Sheline, and E.T. Jurney, Phys. Rev. C 3, 766 (1971). 
UCRL-TR-200316 
TABLE I: Relative $\gamma$-ray intensities for rotational levels in ${ }^{175} \mathrm{Lu}$. Only those levels where new branching-ratio information could be obtained are listed.

\begin{tabular}{|c|c|c|c|c|}
\hline Band & Level (keV) & Spin & $E_{\gamma}$ & $I_{\gamma}^{\text {rel }}$ \\
\hline \multirow[t]{7}{*}{$1 / 2^{-}[541]$} & 514.5 & $3 / 2^{-}$ & 143.8 & $35(6)$ \\
\hline & & & 161.2 & 100 \\
\hline & 672.5 & $7 / 2^{-}$ & 257.8 & $94(11)$ \\
\hline & & & 319.1 & 100 \\
\hline & 885.9 & $11 / 2^{-}$ & 213.5 & $17(3)$ \\
\hline & & & 323.4 & $92(12)$ \\
\hline & & & 471.1 & 100 \\
\hline \multirow[t]{8}{*}{$9 / 2^{-}[514]$} & 685.5 & $13 / 2^{-}$ & 156.1 & 100 \\
\hline & & & 289.1 & $15(2)$ \\
\hline & 864.2 & $15 / 2^{-}$ & 178.8 & 100 \\
\hline & & & 334.8 & $33(4)$ \\
\hline & 1064.6 & $17 / 2^{-}$ & 200.4 & 100 \\
\hline & & & 379.1 & $41(6)$ \\
\hline & 1285.5 & $19 / 2^{-}$ & 220.9 & 100 \\
\hline & & & 421.3 & $117(22)$ \\
\hline \multirow[t]{4}{*}{$7 / 2^{-}[523]$} & 1112.3 & $9 / 2^{-}$ & 582.8 & 100 \\
\hline & & & 716.1 & $27(5)$ \\
\hline & 1242.4 & $11 / 2^{-}$ & 556.8 & 100 \\
\hline & & & 713.1 & $69(12)$ \\
\hline \multirow[t]{14}{*}{$1 / 2^{+}[411]$} & 632.3 & $3 / 2^{+}$ & 261.8 & $23(3)$ \\
\hline & & & 279.1 & $38(4)$ \\
\hline & & & 289.2 & 100 \\
\hline & 757.0 & $5 / 2^{+}$ & 124.6 & 100 \\
\hline & & & 130.9 & $15(3)$ \\
\hline & 773.0 & $7 / 2^{+}$ & 140.5 & 100 \\
\hline & & & 226.4 & $19(3)$ \\
\hline & & & 340.7 & $46(6)$ \\
\hline & & & 413.7 & $13(2)$ \\
\hline & & & 420.1 & $28(4)$ \\
\hline & 989.7 & $9 / 2^{+}$ & 216.6 & 100 \\
\hline & & & 232.7 & $70(9)$ \\
\hline & 1316.8 & $13 / 2^{+}$ & 297.9 & $50(8)$ \\
\hline & & & 327.2 & 100 \\
\hline \multirow[t]{4}{*}{$3 / 2^{+}[411]$} & 1149.9 & $3 / 2^{+}$ & 517.5 & 100 \\
\hline & & & 523.7 & $93(14)$ \\
\hline & 1218.4 & $5 / 2^{+}$ & 461.5 & $87(13)$ \\
\hline & & & 585.9 & 100 \\
\hline
\end{tabular}




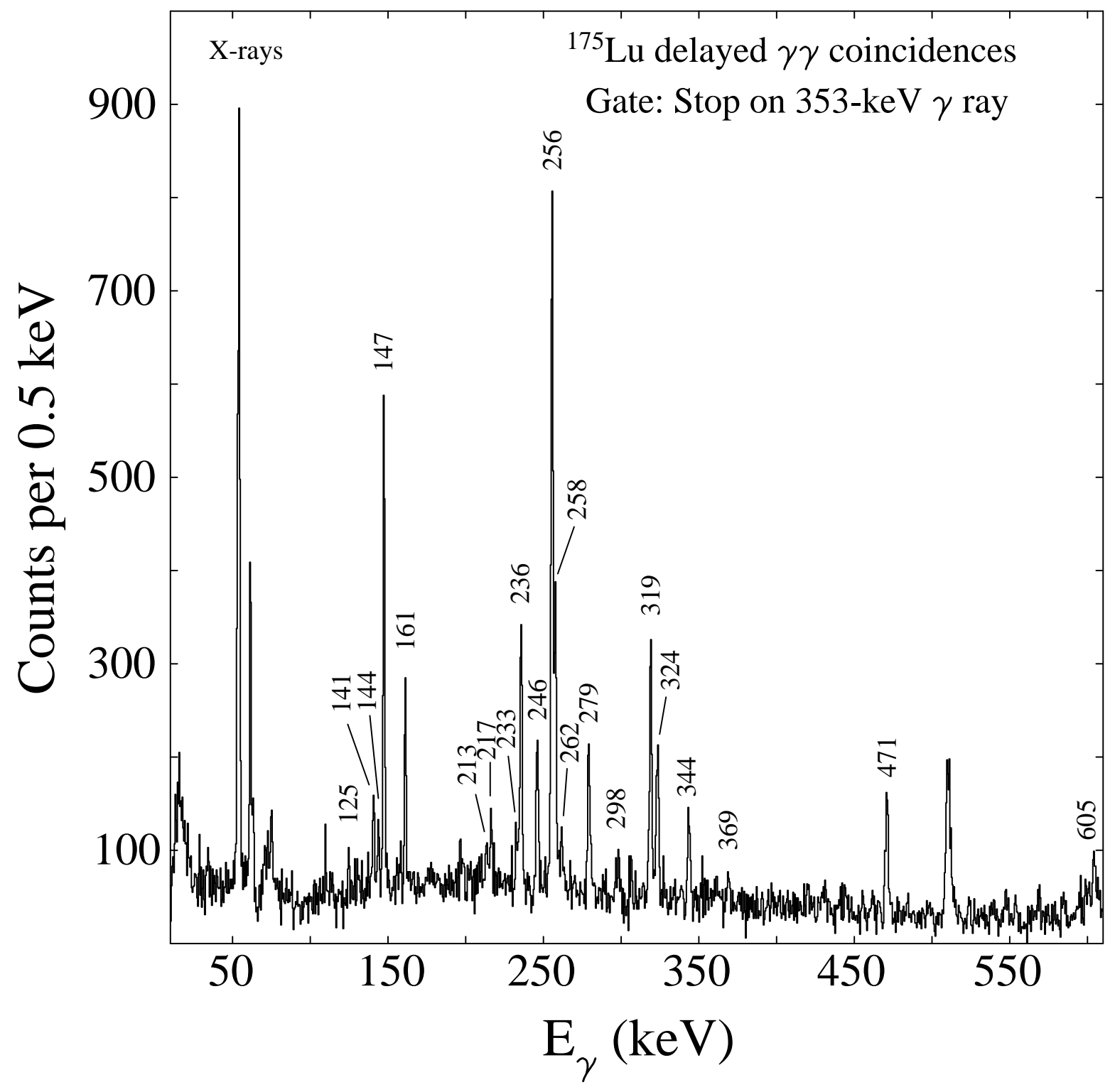

FIG. 1: Spectrum observed for a gate taken on the 353-keV transition serving as the "stop" transition for delayed $\gamma \gamma$ coincidences. The $\gamma$ rays placed above the 1.49(7) $\mu$ s $353-\mathrm{keV}$ level in ${ }^{175} \mathrm{Lu}$ are labeled with their energies in $\mathrm{keV}$. 
UCRL-TR-200316

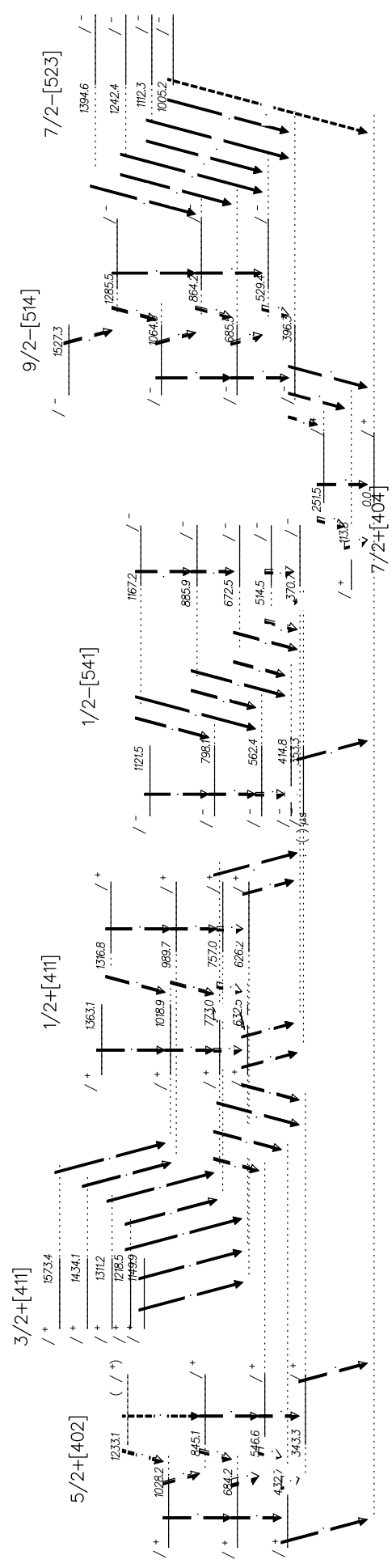

FIG. 2: Partial level scheme leading to new results in ${ }^{175} \mathrm{Lu}$ constructed from analysis of prompt and delayed $\gamma \gamma$ coincidence. The $6-\mathrm{keV} 3 / 2^{+} \rightarrow 1 / 2^{+}$and $16-\mathrm{keV} 7 / 2^{+} \rightarrow 5 / 2^{+}$ transitions in the $1 / 2^{+}[411]$ band are placed due to the observation of cross-talk between the two signatures in the prompt-coincidence spectra. The $17-\mathrm{keV} 1 / 2^{-} \rightarrow 5 / 2^{-}$transition in the $1 / 2^{-}$[541] band is placed due to the observation of the $144-\mathrm{keV}$ transition in the $353-\mathrm{keV}$ delayed coincidence gate (see Fig.1). Uncertainties on the $\gamma$-ray energies are estimated at $0.1 \mathrm{keV}$. 


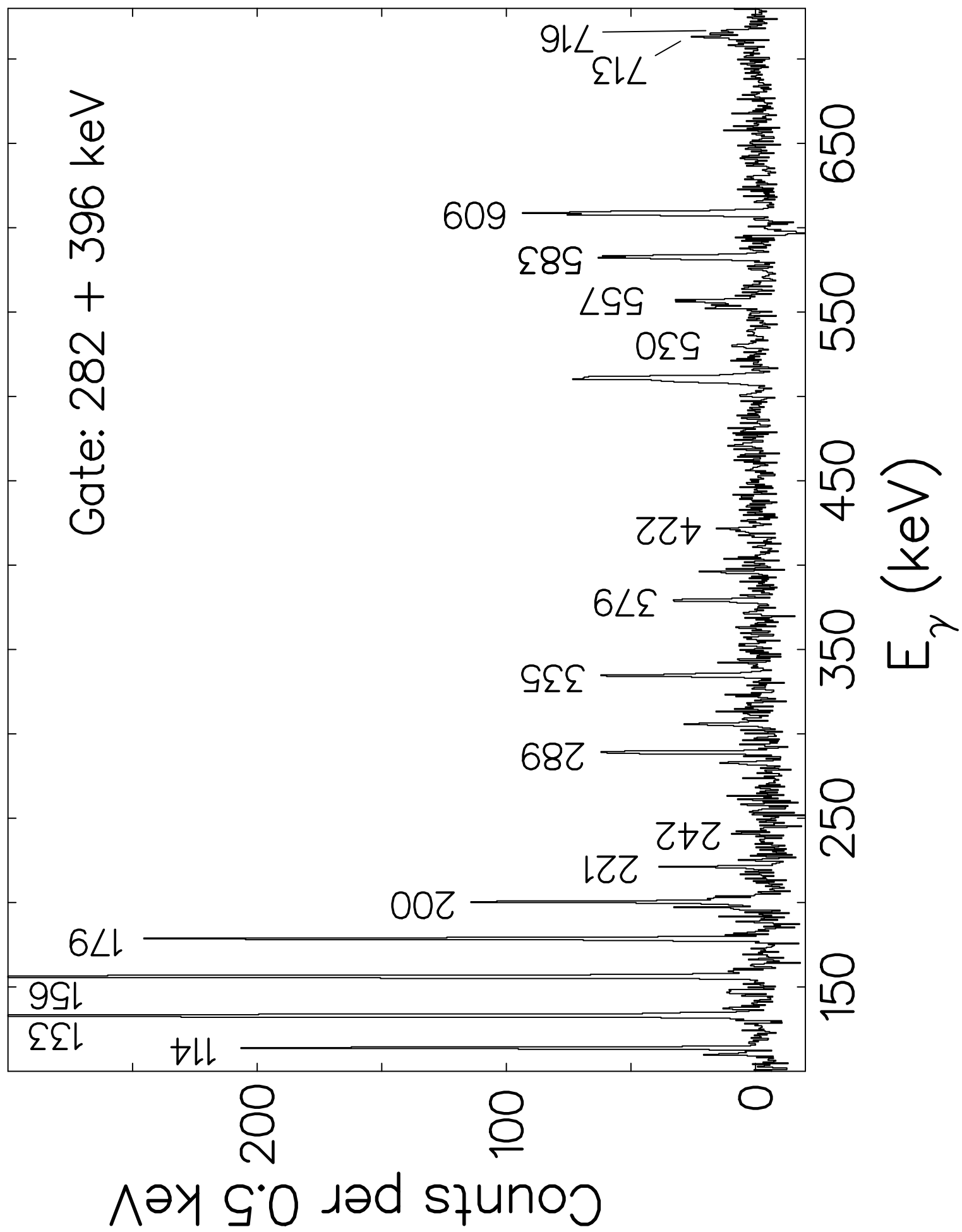

FIG. 3: Spectrum obtained from the prompt $\gamma \gamma$ coincidences with a sum of gates taken on the $282 \mathrm{keV}$ and $396-\mathrm{keV}$ transitions. 


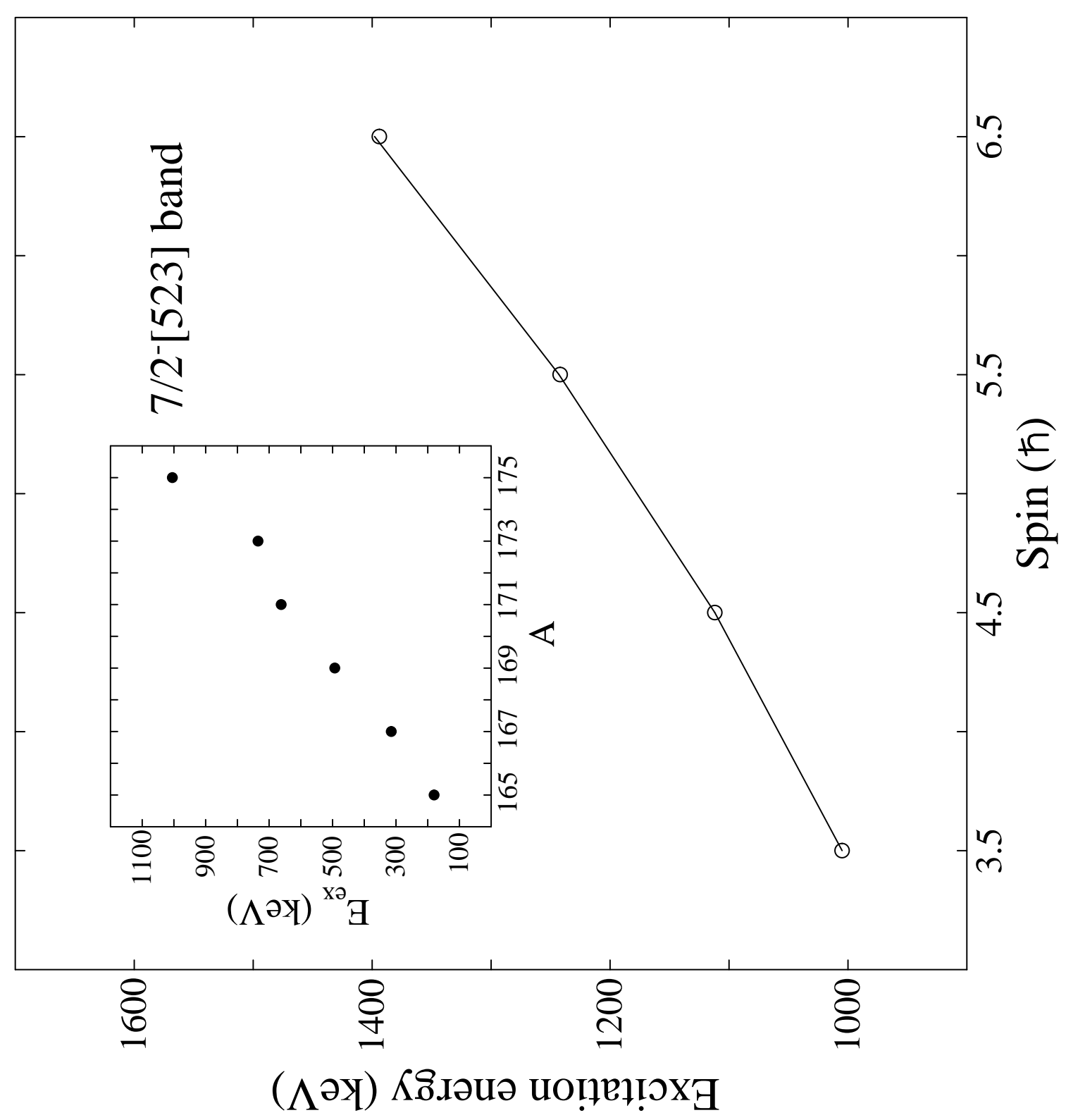

FIG. 4: Fit to proposed members of the $7 / 2^{-}[523]$ band. The data points are the observed level energies, and the line is the fit using a rotational formula with $K=7 / 2$ and a rotational parameter of $11.9 \mathrm{keV}$. The inset shows the experimental band heads of the $7 / 2^{-}[523]$ configuration, where known, in the $\mathrm{Lu}$ isotopes. 
UCRL-TR-200316

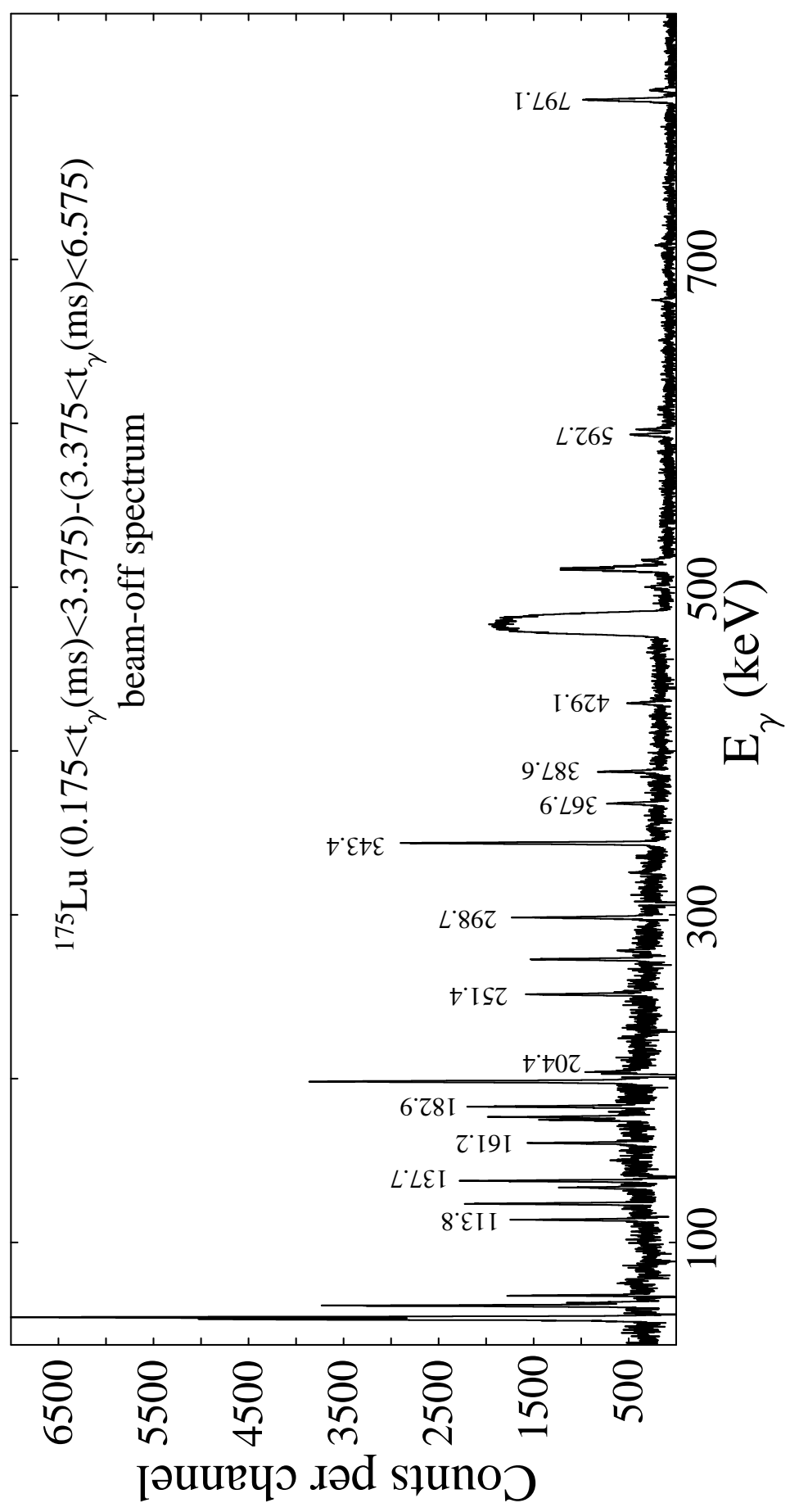

FIG. 5: Spectrum obtained from the beam-off period by subtracting events with a time gate of $3.375<t_{\gamma}(\mathrm{ms})<6.575$ from that with a time gate of $0.175<t_{\gamma}(\mathrm{ms})<3.375$. The $\gamma$ rays belonging to the decay path of the $K^{\pi}=19 / 2^{+}$isomer are labeled with their energies in $\mathrm{keV}$. 
UCRL-TR-200316

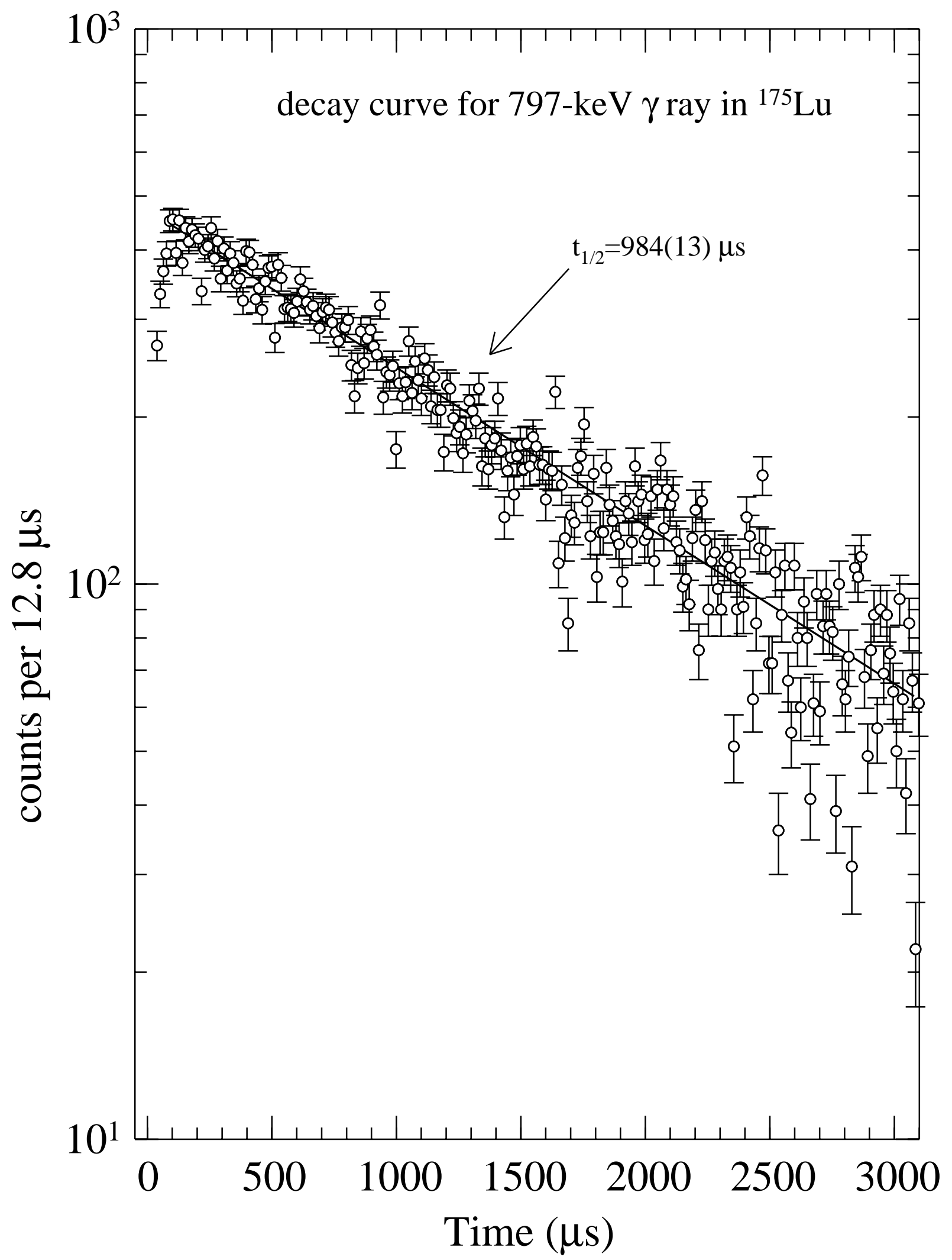

FIG. 6: Decay curve obtained for the $797-\mathrm{keV}$ transition depopulating the $K^{\pi}=19 / 2^{+}$ isomer. A time-dependent dead time correction has been applied to the data. The fit yields a half life of $984 \pm 13$ (stat.) \pm 30 (sys.) $\mu$ s. 